\title{
Food for Thought: Dietary Intervention in a Rare Cause of Severe Ventilatory Failure
}

\author{
Henry Yung ${ }^{1,2}$, Keshav Sharma ${ }^{1}$, William Flowers ${ }^{1}$, Malcolm Marquette ${ }^{1}$, \\ Laura Starace $^{3}$, Clare Sander ${ }^{1}$, Rowan Burnstein ${ }^{4}$, Jurgen Herre ${ }^{1}$ \\ ${ }^{1}$ Respiratory Department, Cambridge University Hospitals, Cambridge, UK \\ ${ }^{2}$ Rayne Institute, University College London, London, UK \\ ${ }^{3}$ Nutrition and Dietetics Team, Cambridge University Hospitals, Cambridge, UK \\ ${ }^{4}$ Neurocritical Care Unit, Cambridge University Hospitals, Cambridge, UK
}

Received: 26/01/2020

Accepted: $18 / 02 / 2020$

Published: 26/03/2020

How to cite this article: Yung H, Sharma K, Flowers W, Marquette M, Starace L, Sander C, Burnstein R, Herre J. Food for thought: dietary intervention in a are cause of ventilatory failure. EJCRIM 2020;7: doi:10.12890/2020_001523.

Conflicts of Interests: The Authors declare that there are no competing interests.

Acknowledgements: The authors would like to thank Dr Penny Wright and Dr Adam Duckworth for provision of histology images, and also the patient for consenting to the publication of this case.

This article is licensed under a Commons Attribution Non-Commercial 4.0 License

\section{ABSTRACT}

Plastic bronchitis is a rare condition characterised by endobronchial cast formation. We report the case of a 53-year-old women who deteriorated following an elective bronchoscopy procedure. She developed refractory ventilatory failure and required repeated bronchoscopy, which identified thick tenacious casts as the cause of her airway compromise. She did not respond to conventional therapies including endoscopic clearance, mucolytic therapy and nebulised tissue plasminogen activator (TPA). Total parenteral nutrition and a fat-free enteral diet were instituted while the patient was on extracorporeal membrane oxygenation (ECMO), which led to substantial improvement in her condition and demonstrated the importance of dietary strategies in this case.

\section{LEARNING POINTS}

- Plastic bronchitis can rarely present in adults with acute ventilatory failure and life-threatening airway obstruction.

- Although there are no established guidelines on management, dietary intervention (e.g., a fat-free diet) should be strongly considered as a therapeutic option.

- Extracorporeal membrane oxygenation (ECMO) is feasible and should be considered in plastic bronchitis with airway compromise.

\section{KEYWORDS}

Plastic bronchitis, bronchial casts, extracorporeal membrane oxygenation, asthma, mechanical ventilation

\section{INTRODUCTION}

Plastic bronchitis is an uncommon condition characterised by endobronchial cast formation ${ }^{[1]}$. Alternative descriptors include cast bronchitis, Hoffman's bronchitis, fibrinous bronchitis and pseudomembranous bronchitis ${ }^{[2]}$. It was first described by Galen in the second century AD as 'venae arteriosae expectorantii', translated as 'expectorated arteries and veins'. Proposed mechanisms include the inflammatory response in asthma, raised pulmonary venous pressures after the Fontan procedure, and lymphatic abnormality leading to chyle leakage. 


\section{CASE DESCRIPTION}

A 53-year-old woman was referred to the chest clinic with a 3-month history of expectoration of bronchial casts. She had been diagnosed with asthma 21 years previously and managed with Becotide and Ventolin inhalers. Her medical history included polycystic ovary syndrome, hirsutism, type 2 diabetes mellitus and obesity. Since the 1990s, she had had recurrent bouts of pericarditis with normal coronary angiogram and cardiac MRI findings. She was previously investigated for arthralgia, fatigue, bilateral anterior uveitis and oral ulcers with no genital involvement. Behçet's disease had been suggested but investigations for this had been negative.

In the clinic, she reported occasional chest tightness and dyspnoea with no wheeze. She produced thick sputum, resembling casts, 10-12 times per day. She was a never smoker. Serological investigations including IgE, specific IgE for Aspergillus fumigatus and ANCA were unremarkable. Historical eosinophil counts were all within the normal range. Lung function tests showed a minimal obstructive defect: forced expiratory volume 1 second $2.13 \mathrm{~L}(88.2 \%$ ), forced vital capacity $2.89 \mathrm{~L}$ (101.6\% predicted) and forced expiratory ratio $67.2 \%$, with normal lung volumes and gas transfer. Peak flow ranged from 350 to $400 \mathrm{~L} / \mathrm{min}$ with minimal diurnal variation. Baseline computed tomography (CT) of the chest revealed moderate air trapping in keeping with small airway disease. Bronchoscopy was performed and showed no endobronchial lesions, secretions or abnormal airway inflammation. Routine endobronchial mucosal biopsies were taken for histopathology.

\section{Inpatient Admission}

The patient was readmitted the day following bronchoscopy with cough, dyspnoea and pyrexia. Chest radiography showed evidence of right lower lobe consolidation (Fig. 1). Intravenous ciprofloxacin and metronidazole were commenced, followed by vancomycin and hydrocortisone. A bedside echocardiogram showed no significant biventricular dilatation or impairment. The patient deteriorated despite high flow oxygen and non-invasive ventilatory support, and required mechanical ventilation 6 days following admission.

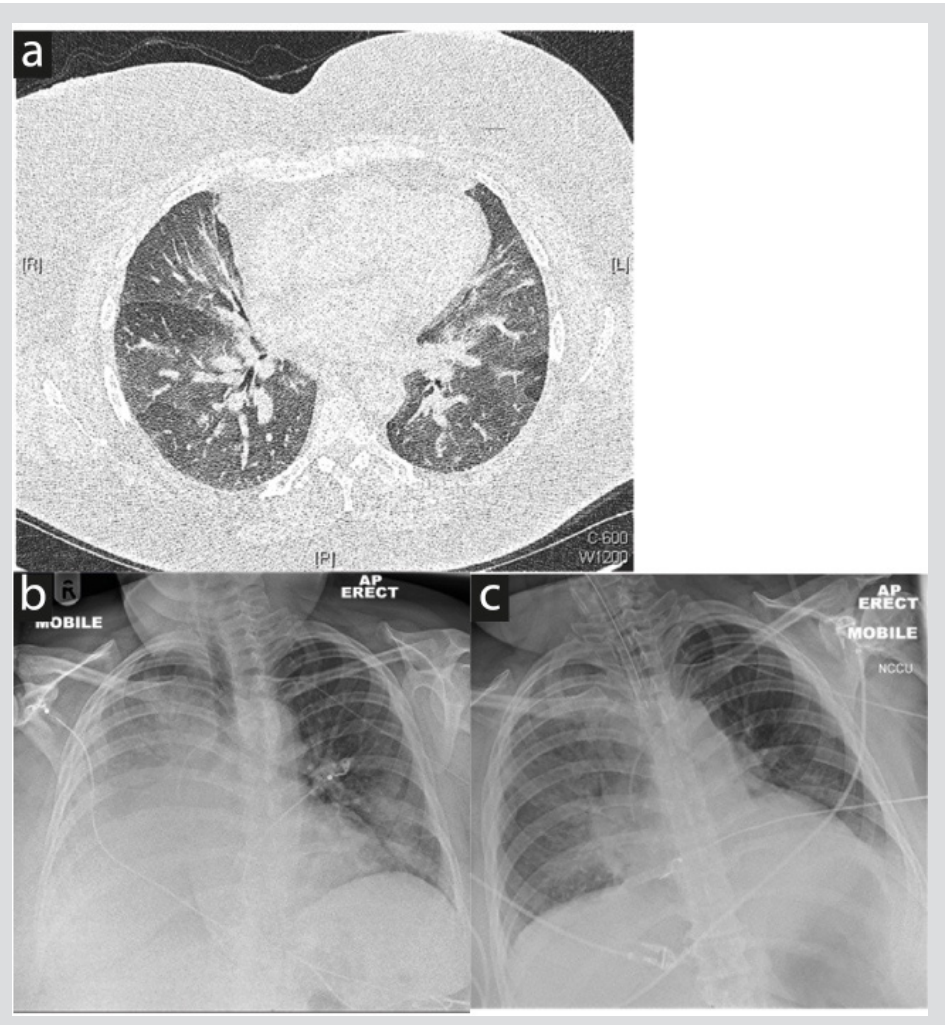

Figure 1 (a) Baseline CT image showed evidence of expiratory air trapping indicative of small airway disease. (b) Chest radiograph during early admission showed right lower lobe collapse and consolidation. (c) Resolution of radiographic changes following bronchoscopic airway clearance.

Broncho-alveolar lavage cultures grew mixed respiratory flora. Respiratory viral PCR, an HIV screen and urinary legionella antibody were negative. There was delayed detection of Pneumocystis jirovecii (cycle 33) likely representing colonisation. Antibiotics were changed to meropenem and clarithromycin. Ventilation was difficult due to to fibrinous casts plugging her airways. Repeated bronchoscopies revealed thick tenacious casts that were removed with a biopsy forceps and large-volume saline flushes (Figs. 2 and 3). There was no evidence of underlying airway injury or stenoses. Returned fluid appeared chylous in nature reflecting possible lymphatic pathology. 


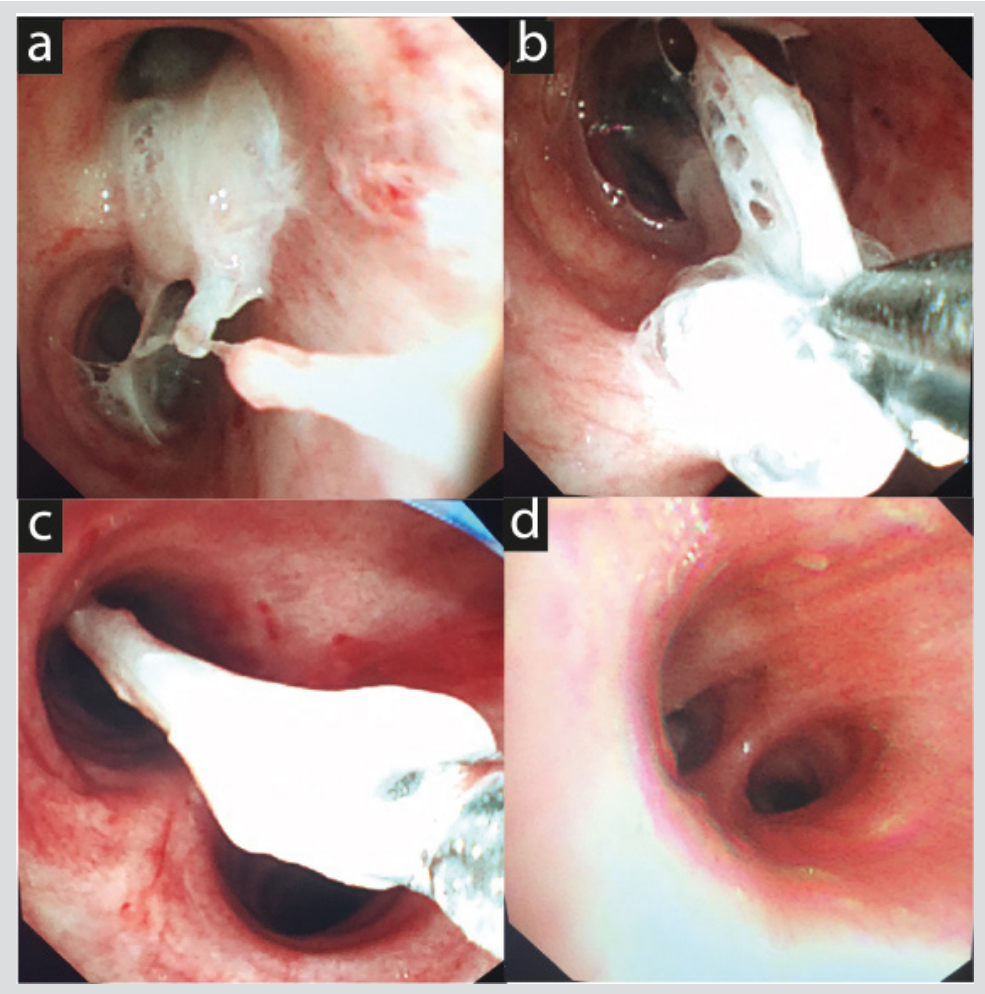

Figure 2 (a-c) Bronchoscopy showed thick white tenacious plugs obstructing the right lower lobe, which required removal with a biopsy forceps. (d) Repeat procedure shows remarkable improvement with some residual granulat on tissue in the lower lobes.

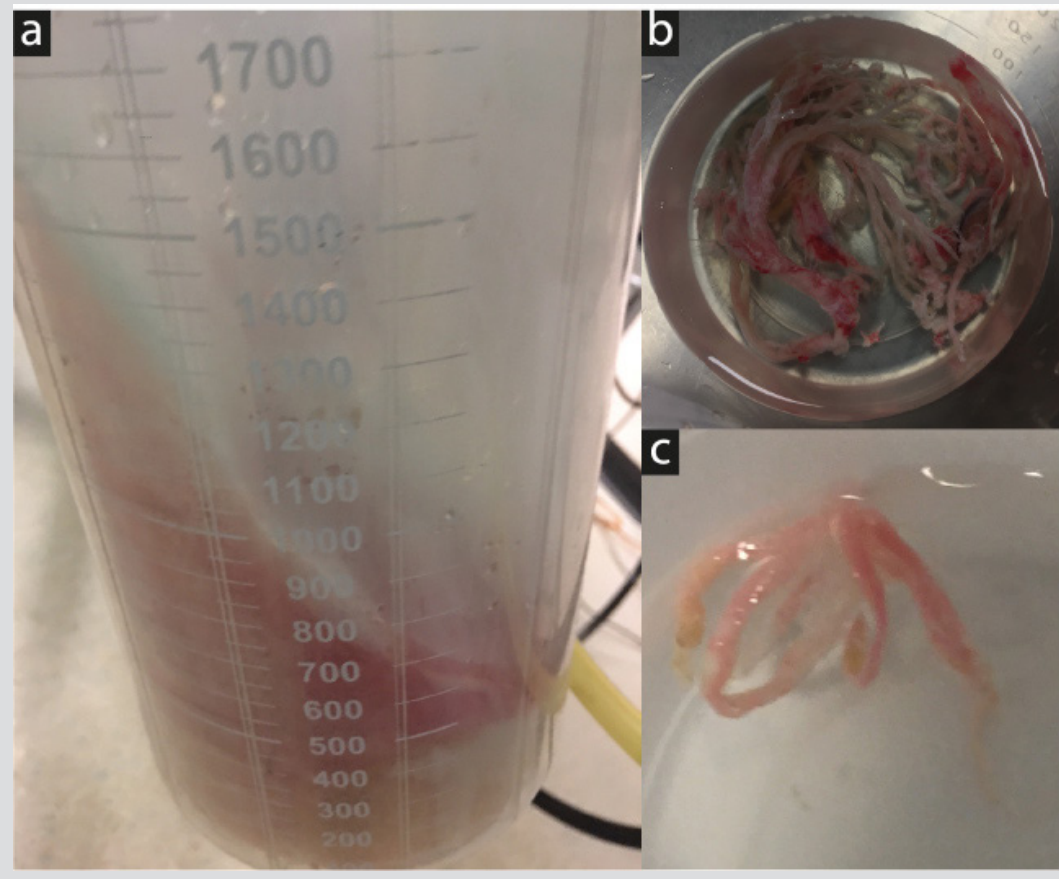

Figure 3 (a) Bronchial lavage fluid showed a white opaque layer suggesting it may have a chylous nature. (b) Bronchial casts in the form of aggregated segments resembling bronchial wall anatomy. (c) Close-up of a single bronchial cast

Nebulised acetylcysteine, saline and tissue plasminogen activator (TPA) were trialled. Human albumin solution was given experimentally to reduce fluid diffusion into the lungs. The patient persistently required high inspired concentrations of oxygen (80-100\%), and was ventilated with low tidal volumes (4-5 ml/kg) and high levels of PEEP (up to $12 \mathrm{~cm} \mathrm{H}_{2} \mathrm{O}$ ). Despite conventional treatment, the patient had several periarrest episodes due to inadequate ventilation, and veno-venous ECMO (VV ECMO) was commenced on day 14.

Initially, the patient required twice daily bronchoscopic clearance of casts. Radiological embolization of the thoracic duct or surgical ligation was considered, but her clinical condition was too unstable for this to be performed safely. A fat-free enteral feed was commenced in the interim, and sedation changed from propofol to midazolam to reduce chyle production. 
The patient's respiratory status gradually improved with reduced need for airway clearance. A tracheostomy was performed on day 27 and she came off VV ECMO on day 32. She was sent back to our local centre with minimal oxygen requirements and pressure support. She was eventually weaned off the ventilator and decannulated after 45 days. She has been discharged and remains stable on a fat-free diet.

\section{DISCUSSION}

As this patient was deteriorating despite optimal supportive management, we were compelled to consider alternative treatments that may save her life. The chylous nature of the return broncho-alveolar lavage fluid; lymphocytic predominance in casts (Fig. 4); and short interval from mucosal biopsy to admission suggested abnormal leakage of chyle from damaged epithelium. This is likely the aggravating factor for her decline, if not the underlying aetiology. Indeed, it has been hypothesised that low grade trauma may cause rupture of superficial lymphatic vessels in plastic bronchitis, leading to seepage of lymph into the bronchial lumen ${ }^{[3]}$. We therefore hypothesised that lowering fat intake would reduce chyle flow through damaged lymph vessels and ameliorate the situation.

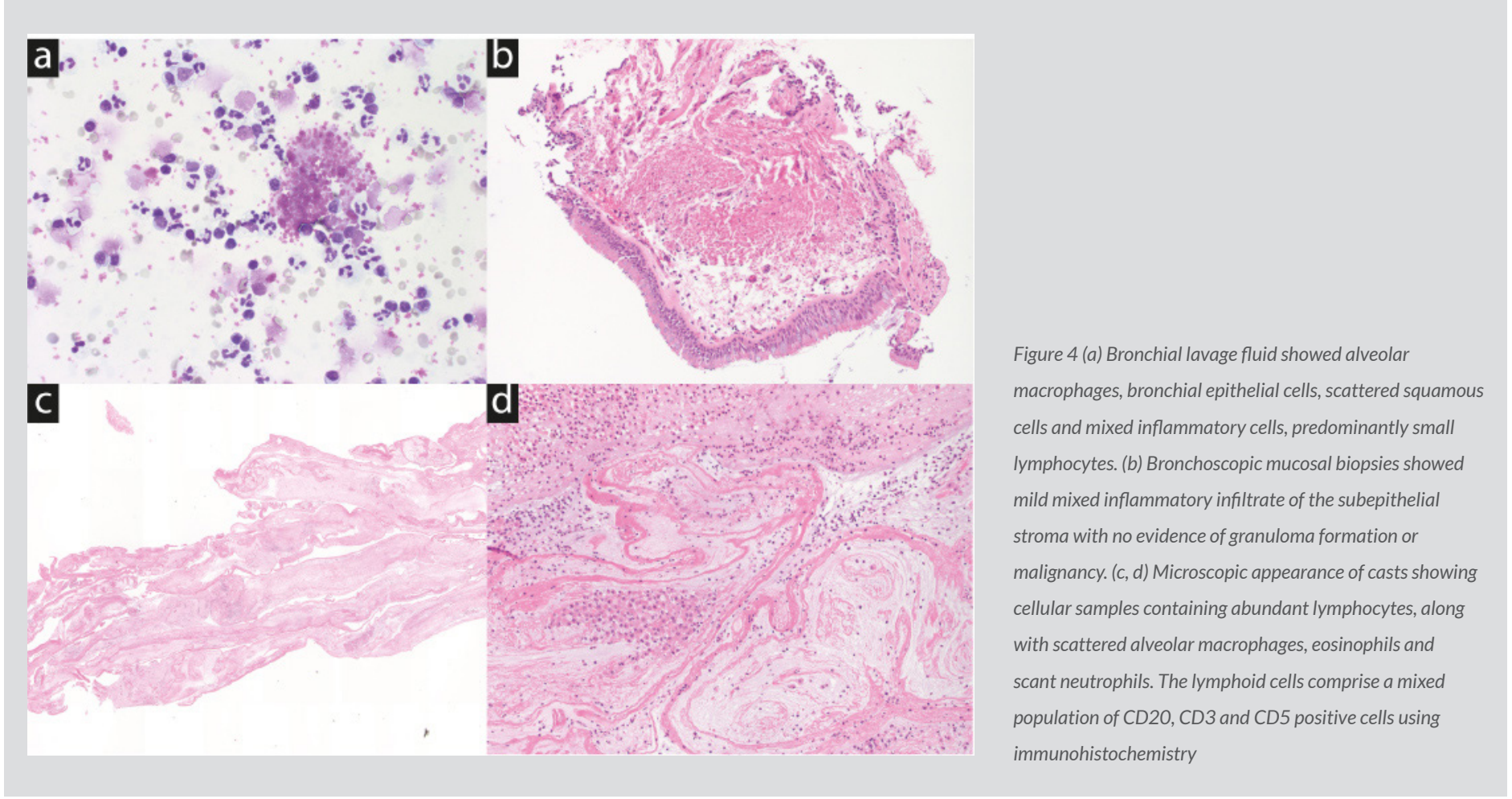

We attribute the successful outcome in our patient to dietary strategies that reduced lymphatic outflow, and to ECMO used as a bridge while these effects were taking place. Similar anecdotal evidence exists for the management of other chylous disorders (e.g. chylothorax). There are no specific dietary guidelines for the management of plastic bronchitis, and the dietary regimen was chosen following input from our dietician colleagues. Parenteral nutrition is an alternative method that prevents enteral fat absorption. Other pharmacological interventions reported in the literature include inhaled recombinant DNase, octreotide and rapamycin inhibitor ${ }^{[4]}$. If conservative measures fail, lymphangiography and embolization, or video-assisted thoracoscopic thoracic duct ligation may be effective in some cases ${ }^{[5]}$.

\section{REFERENCES}

1. Cavric G, Naumovski-Mihalic S, Kardum-Skelin I, et al. [Acute plastic bronchitis--case report]. Acta Med Croatica 2011;65(Suppl 1):127-131.

2. Turgut T, In E, Ozercan IH, et al. A case of plastic bronchitis. Arch Iran Med 2014;17(8):589-590.

3. Itkin M. Interventional treatment of pulmonary lymphatic anomalies. Tech Vasc Interv Radiol 2016;19(4):299-304.

4. Shah A, Donovan J, Marino P, et al. A lesson in plasticity: a 74-year-old man with plastic bronchitis. Thorax 2017;72(11):1055-1057.

5. Guevara CJ, Rialon KL, Ramaswamy RS, et al. US-guided, direct puncture retrograde thoracic duct access, lymphangiography, and embolization: feasibility and efficacy. J Vasc Interv Radiol 2016;27(12):1890-1896. 